\title{
NOTE
}

\section{Amino Acid and Vitamin Requirements of Micrococcus Species Isolated from Human Skin}

\author{
JULIAN W. FARRIOR AND WESLEY E. KLOOS \\ Department of Genetics, North Carolina State University, Raleigh, North Carolina 27607
}

\begin{abstract}
Amino acid and vitamin requirements were determined for 215 natural auxotrophic strains of micrococci isolated from human skin. Requirement parameters were distinctly different for each of the seven Micrococcus species studied.
\end{abstract}

Kloos, Tornabene, and Schleifer (4) isolated and characterized micrococci from human skin. These strains were isolated from the healthy skins of a group of 20 people living in Raleigh, N.C., and a group of 115 people from 21 different regions of the United States. The micrococci were divided into seven species by various morphological, physiological, genetic, and biochemical criteria. Several of these species were new or were not previously characterized. A total of 621 strains of these micrococci were tested for growth on a defined inorganic nitrogen agar medium and a complete agar medium containing peptone and yeast extract (8) to identify strains with organic nitrogen requirements (4).

Micrococci exhibiting absolute organic nitrogen requirements or slightly leaky growth on the inorganic nitrogen medium in the above-mentioned study were selected to determine the specific amino acid and vitamin requirements.

These requirements were determined by several criteria: (i) the absence (absolute requirement) or marked reduction (leakiness) of growth by the single deletion of each metabolite from a defined medium containing the amino acids serine, isoleucine, valine, tyrosine, glycine, histidine, alanine, aspartate, threonine, tryptophan, proline, phenylalanine, arginine, lysine, and glutamate (each at $500 \mu \mathrm{g} / \mathrm{ml}$ ), cysteine, methionine, and leucine (each at 100 $\mu \mathrm{g} / \mathrm{ml}$ ), and the vitamins thiamine, niacin, riboflavin, pantothenic acid, and folic acid (each at $1.2 \mu \mathrm{g} / \mathrm{ml}$ ), and biotin (at $0.025 \mu \mathrm{g} / \mathrm{ml}$ ); (ii) the presence of growth by the single addition of a metabolite from part (i) above to the inorganic nitrogen medium in those strains requiring only one metabolite; (iii) the presence of growth by the addition of the required metabolites as determined in part (i) to the inorganic nitrogen medium in those strains requiring more than one metabolite. In this nutritional study, colony size (diameter) rather than optical density was used as a measure of growth. This procedure is expedient and is commonly employed in studies of nutritional requirements of mutagen-induced auxotrophs and in genetic studies where it is necessary to visualize a significant portion of the population. Colony size correlated well with optical density measurements and was used here mainly to explore the qualitative identification of natural auxotrophs.

The Micrococcus species showed distinctly different parameters for amino acid and vitamin requirements and thus further supported their taxonomic separation based on various other criteria (4-7). The amino acid requirements are shown in Fig. 1 and vitamin requirements are shown in Fig. 2.M. luteus auxotrophs were usually leaky and only a few strains showed absolute requirements. These requirements were usually for cysteine, methionine, or tyrosine. Natural auxotrophs of $M$. luteus in this study did not require a purine as reported for old laboratory strains $(2,3) . M$. lylae, which is closely related to the species $M$. luteus (4), required methionine and multiple vitamins. The marked difference in nutritional requirements between $M$. luteus and $M$. lylae further supports their separation into different species. $M$. sedentarius appeared to have multiple amino acid requirements, and pantothenic acid was the major vitamin required. Many strains of $M$. varians had nonspecific amino acid requirements, i.e., several different amino acids can apparently satisfy the organic nitrogen requirement; however, some strains specifically required methionine. Vitamin requirements of this species included thiamine, 

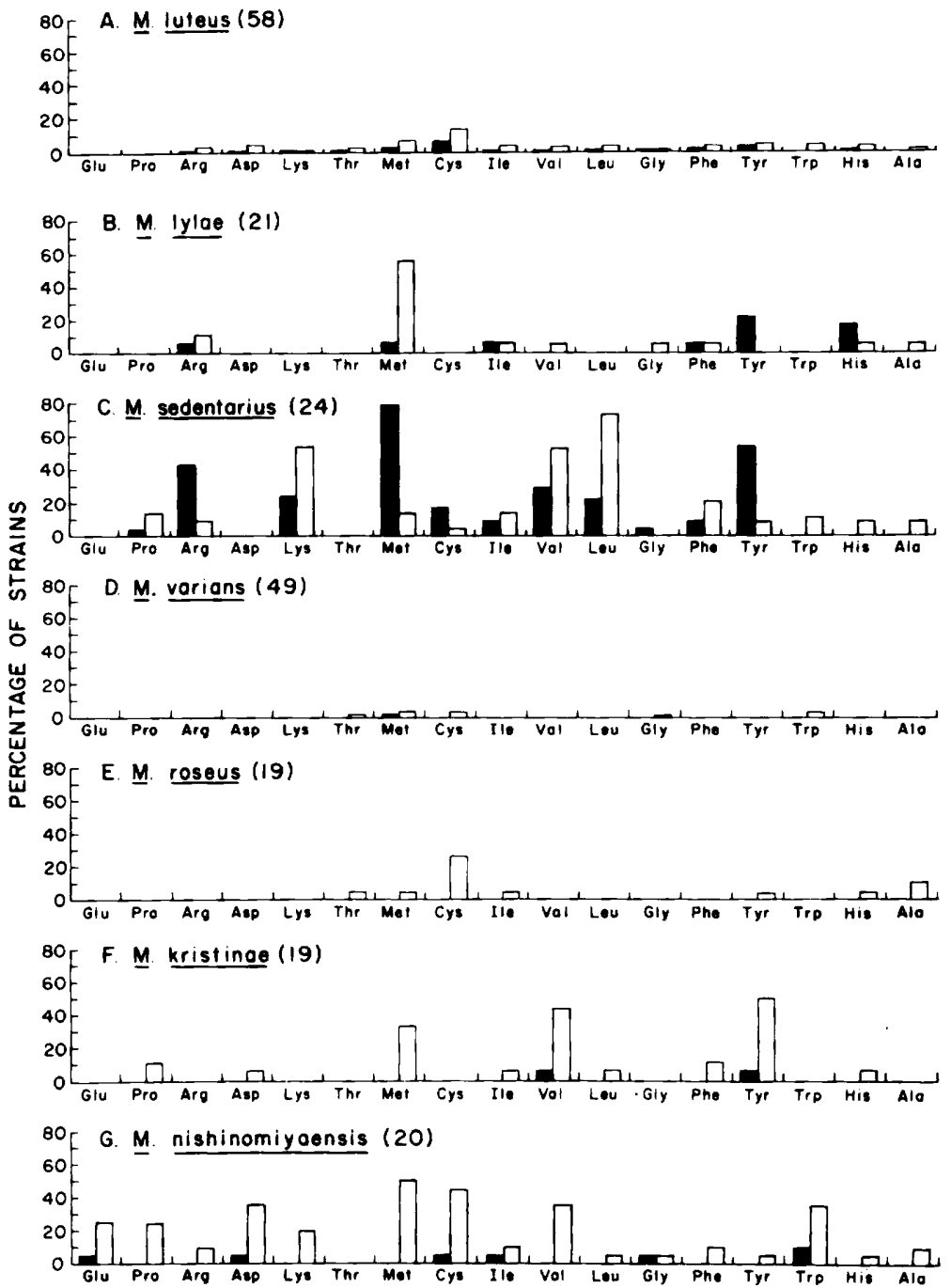

AMINO ACIO REQUIREMENT

FIG. 1. Amino acid requirements of micrococci isolated from human skin. The number of strains tested for each species is given in parentheses. The percentage of strains with an absolute requirement is shown by the solid bar, whereas the outlined bar shows the percentage of strains that is slightly leaky. Abbreviations: Glu, glutamate; Pro, proline; Arg, arginine; Asp, aspartate; Lys, lysine; Thr, threonine; Met, methionine; Cys, cysteine; Ile, isoleucine; Val, valine; Leu, leucine; Gly, glycine; Phe, phenylalanine; Tyr, tyrosine; Trp, tryptophan; His, histidine; Ala, alanine.

niacin, or pantothenic acid. $M$. roseus had partial requirements for cysteine and thiamine. Based on cell wall composition and various biochemical and physiological criteria (4-6), M. varians and $M$. roseus appear to be closely related, and their nutritional requirements appear also to be more similar than those between other species. The frequencies of requirements for cysteine, niacin, and pantothenic acid are, however, significantly different between these two species. $M$. kristinae had multiple amino acid requirements and niacin was the primary vitamin required. $M$. nishinomiyaensis had many amino acid requirements, and the vitamin requirements included thiamine, niacin, or pantothenic acid. It is interesting that the development of elaborate multiple amino acid requirements in $M$. sedentarius and $M$. nishinomiyaensis is correlated with certain questionable clues of relationships 

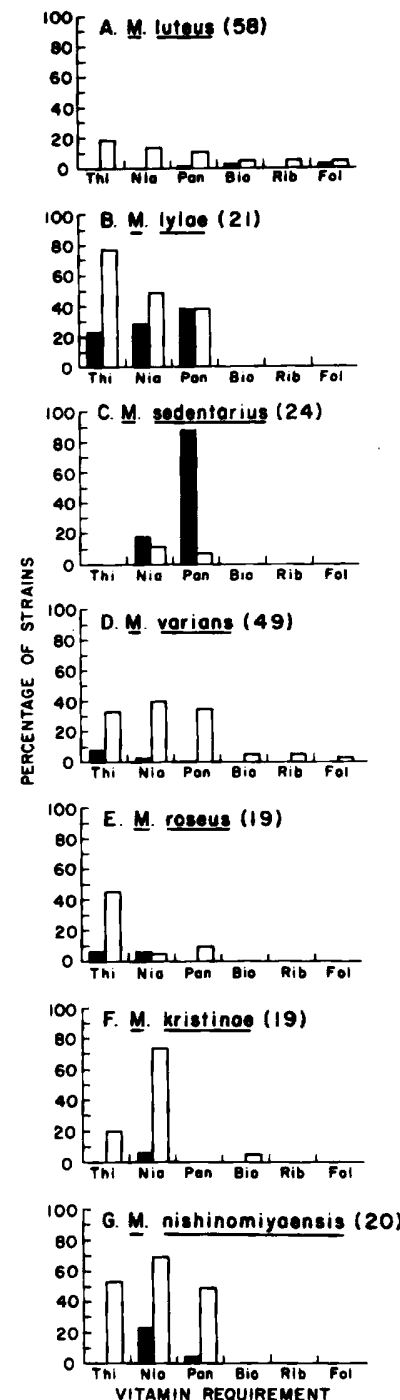

Fig. 2. Vitamin requirements of micrococci isolated from human skin. The number of strains tested for each species is given in parentheses. The percentage of strains with an absolute requirement is shown by the solid bar, whereas the outlined bar shows the percentage of strains that is slightly leaky. Abbreviations: Thi, thiamine; Nia, niacin; Pan, pantothenic acid; Bio, biotin; Rib, riboflavin; Fol, folic acid.

to coryneform bacteria, including similarities of cell wall peptidoglycans and in the sporadic occurrence of aliphatic hydrocarbons, respec- tively (4). Hence, the multiple nutritional requirements may be suggesting a unique relationship of these two species.

Recognizing that methionine and cysteine were required by a large number of the strains, preliminary investigations were directed toward the biosynthetic pathways of these amino acids. Known intermediates in the interrelated cysteine and methionine biosynthetic pathways were tested as growth stimulants (1). Results of responses to the intermediates indicate that there are natural Micrococcus auxotrophs that appear to be blocked in many of the steps that correspond with the Salmonella pathway, thus suggesting that these bacteria share a similar pathway (9).

We thank Margaret Musselwhite for her technical assistance.

This investigation was supported by Public Health Service grant AI 08255 from the National Institute of Allergy and Infectious Diseases.

\section{REPRINT REQUESTS}

Address requests for reprints to: Julian W. Farrior, Department of Genetics, North Carolina State University, Raleigh, N.C. 27607.

\section{LITERATURE CITED}

1. Grabow, W. O. K., and J. A. Smit. 1967. Methionine synthesis in Proteus mirabilis. J. Gen. Microbiol. 46:47-57.

2. Kloos, W. E. 1969. Transformation of Micrococcus lysodeikticus by various members of the family Micrococcaceae. J. Gen. Microbiol. 59:247-255.

3. Kloos, W. E., and L. M. Schultes. 1969. Transformation in Micrococcus lysodeikticus. J. Gen. Microbiol. 55:307-317.

4. Kloos, W. E., T. G. Tornabene, and K. H. Schleifer. 1974. Isolation and characterization of micrococci from human skin, including two new species: Micrococcus lylae and Micrococcus kristinae. Int. J. Syst. Bacteriol. 24:79-101.

5. Kocur, M., and T. Martinec. 1972. Taxonomic status of Micrococcus varians Migula 1900 and designation of the neotype strain. Int. J. Syst. Bacteriol. 22:228-232.

6. Kocur, M., and Z. Pácová. 1970. The taxonomic status of Micrococcus roseus Flügge 1886. Int. J. Syst. Bacteriol. 20:233-240.

7. Kocur, M., Z. Pácová, and T. Martinec, 1972. Taxonomic status of Micrococcus luteus (Schroeter 1872) Cohn 1872 , and designation of the neotype strain. Int. J. Syst. Bacteriol. 22:218-223.

8. Naylor, H. B., and E. Burgi. 1956. Observations on abortive infection of Micrococcus lysodeikticus with bacteriophage. Virology 2:577-593.

9. Smith, D. A. 1971. S-amino acid metabolism and its regulation in Escherichia coli and Salmonella typhimurium. Advan. Genet. 16:141-165. 